\title{
Prevalence Of Osteoporosis And Osteopenia Among Pakistani Pre And Post Menopausal Women
}

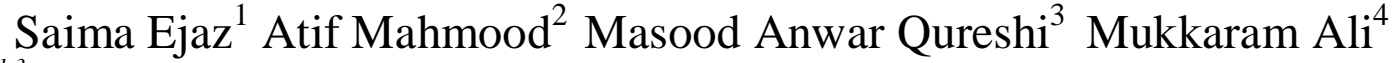 \\ ${ }^{1,3}$ Department of Physiology, Dow International Medical College, Dow University of Health Sciences \\ ${ }^{2}$ Department of Physiology, Shaheed Mohtarma Benazir Bhutto Medical College \\ ${ }^{4}$ Department of Forensic Medicine, Dow International Medical College, Dow University of Health Sciences
}

\begin{abstract}
A cross-sectional study was conducted on 1416 women (age: 25 years or more), belonging to Pre and post-menopausal women (1351 \& 65 respectively). All subjects were interviewed by using a structured Performa for prevalence of risk factors. Bone mineral density (BMD) was measured to determine the degree of Osteoporosis and Osteopenia by speed of sound (SOS) and broad band ultrasound attenuation (BUA) in the calcaneus bone. It was observed that 272 (19.2\%) amongst all subjects were osteoporotic, 890 (62.9\%) osteopenic whereas only 254 (17.9\%) were normal. When assessment was done in groups, it was found that out of the total postmenopausal women (n=65), 32 (49.5\%) were osteoporotic while $28(43.1 \%)$ were found to be osteopenic. On the other hand, in premenopausal women group $(n=1351), 240(17.83 \%)$ were found to be Osteoporotic while $862(63.8 \%)$ were found to be Osteopenic. There was however no significant relation in the BMI of all the three groups. The prevalence of osteoporosis was found to be high in postmenopausal but Osteopenia in premenopausal women was reported to be alarmingly high in premenopausal women probably due to lifestyle and dietary risk factors. There is a need to explore the precise causative factors associated with the high prevalence of Osteopenia recorded in the present study.
\end{abstract}

Key words: Osteoporosis, Osteopenia, Bone Mineral Density, Pre and Post menopausal women

\section{Introduction:}

Osteoporosis and Osteopenia is a major health problem all across the world characterized by low bone mass and density which leads to fragility and increased risk of fracture. High prevalence of Osteoporosis causes vertebral and peripheral fractures that increase morbidity and mortality rate in underdeveloped as well as developed countries ${ }^{[1]}$.

Globally, Osteoporosis is found more in Whites \& Asians and less commonly amongst Blacks. Every year approximately 1.5 million fractures occur which are related to Osteoporosis. These fractures result in permanent disability or death. It is predicted that Osteoporosis-related fracture occur in one out of every two women $\&$ one in every eight men over 50 years of age ${ }^{[2]}$. The direct cost of treatment of hip fracture varies from 4000 to 10,000 USD per case which depends upon the stay at the hospital. Most fractures are treated surgically and the average hospital stay is 7-10 days ${ }^{[3]}$. However, in Asia, it is estimated that by the year 2050, more than about $50 \%$ of all hip fractures will be due to osteoporosis ${ }^{[4]}$.

Pakistan is a third world country and its population is growing rapidly. There is a steady rise in the proportion of elderly population in the country. It is estimated that Pakistani population will reach 226 million till 2020, of which, approximately 16 million people $(7.1 \%)$ will be over 60 years of age ${ }^{[3]}$. Longer life span, low physical activity, diet deficient in calcium and vitamin $\mathrm{D}$ and rich in protein, early menopause and prolonged immobility are some of the major risk factors that make Pre and Post menopausal women prone to the risk of developing Osteoporosis and Osteopenia ${ }^{[5]}$.

International Osteoporosis Foundation conducted a survey on Pakistani population in 2009. The findings of this study were based on ultrasound investigations. It was found that 40 million people ranging between the age group 45-70 years were Osteopenic while almost 10 million people were found to be Osteoporotic. The overall prevalence of Osteoporosis was found to be $16 \%$ while that of Osteopenia was found to be $34 \%{ }^{[6]}$.

No study determining the true prevalence of Osteopenia and Osteoporosis in Pre- and Post-menopausal women of Pakistan has been done so far. Because of this, no epidemiologic survey that has been conducted till now will tell the exact prevalence of Osteoporosis and Osteopenia in Pakistani women. Although such an informative data will prove to be valuable in the prevention and control of an important public health issue.

The key index for diagnosis of Osteopenia and Osteoporosis is Bone Mineral Density, that is amount of bone per meter square, can be measured by Dual Energy X-ray Absorptiometry (DEXA). Although, it is considered to be the gold standard test but its high cost limits its use for community based studies. Quantitative Ultrasound Measurement (QUS) is a technique that is cost effective and estimates calcaneal BMD by attenuation and speed of sound that pass through the heel ${ }^{[7]}$. 


\section{Material And Method:}

A cross sectional survey was conducted at several different outpatient clinics in Karachi, Pakistan from Jan 2009 to Dec 2010. Posters were displayed in the clinics a week before the test was scheduled in order to invite maximum number of participants on the day of the examination.

On the day when the test was to be performed, the procedure was explained to the participants, an informed consent was obtained from each subject and complete confidentiality of the research findings was ensured.

The project was approved by the Institutional Review Board (IRB) of DUHS. A total of 1416 subjects were included in the study, out of which 65 women were Post menopausal and 1351 were Premenopausal. Relevant medical history and physical examination was done and the findings were recorded on a detailed questionnaire. The patients with current or past history of Diabetes Mellitus, Hyperparathyroidism, Renal disorders, Gastrectomy, Rheumatoid Arthritis, Chronic Malabsorption Syndromes, Chronic Liver Disease and also those who had a current or past treatment history with glucocorticoids and/or thyroid hormone were excluded from the study. Subjects with history of any tobacco usage were also excluded.

A food frequency questionnaire was also administered to patients for measurement of daily calcium intake, which was estimated by multiplying the frequency of consumption of calcium rich food (such as dairy products, Almonds, Fish, Spanish, Broccoli and etc) by the calcium content of age specific portion sizes.

Moreover, 30 minute or more work against gravity (like walking, stair climbing and weight training) per day was considered in exercise doing by subjects.

\subsection{Anthropometric Measurement:}

BMI was measured by using the formula Weight in kg divided by Height in meter ${ }^{2}$ The height and weight required for the calculation of the BMI were recorded by using a bathroom weight measuring scale

\subsection{BMD Measurement:}

Bone Mineral density was measured by Bone Densitometer (SONOST 3000). It is small portable device with dry gel system that measures Broadband Ultrasound Attenuation (BUA) in $\mathrm{dB} / \mathrm{MHz}$ and Speed of Sound (SOS). An ultrasound beam is passed through heel. The calcaneus BMD is calculated (in $\mathrm{g} / \mathrm{cm}^{2}$ ) by combination of BUA and SOS which yields the QUI index.

The machine was calibrated and standardized before usage. The test was performed by a trained technician ${ }^{[8]}$. Measurements were taken on the right calcaneus bone of all the subjects. In case of any right-heel fracture or any other disorders, the left heel was examined for measurement.

To evaluate BMD and risk of Osteoporosis and Osteopenia, T scores were used. According to WHO criteria, $\mathrm{T}$ score greater than -1 shows normal BMD, less than -2.5 shows Osteoporosis whereas $\mathrm{T}$ scores in between -1 and -2.5 shows Osteopenic condition. ${ }^{[8,9]}$

DEXA still remains the gold standard for the measurement of BMD but for the indirect assessment of bone quality, DEXA has been widely replaced by Quantitative Ultrasonography (QUS) because of low cost, portability and no ionizing exposure to the patients.

\subsection{Calibration:}

Machine was calibrated and precision was measured before usage to reduce a margin of error and to get accurate value in times of examining bone density ${ }^{[8]}$.

It is to raise credibility of examination results through checking of system reliability on a daily basis throughout study.

To calibrate the system,

1. <DailyTest $>$ button was clicked in the "Home" screen. Then the "DailyTest" screen appears.

2. <StartDailyTest>button was clicked, which starts daily test, after completion, the message box "Complete Dailytest" appears and clicked back to "Home" screen.

3. The selection of "Time" tap, shows graph in the time domain and selection of "Frequency" tap, shows graph on the frequency domain.

4. For calibrating the system QC Phantomis used included in the accessories list.

5. Single click on <Start>button on the bottom, generates ultrasonic waves and the word on the <Start> button is changed into $\langle$ Stop $>$.

6. The message box "Complete Dailytest" appears with an alarm sound after progressing calibration of the system and then $<\mathrm{OK}>$ button is clicked, the progress of calibration is completed. 


\section{Results:}

All the participants were residents of Karachi, Pakistan with Asian ethnicity. The difference in the mean age of the normal (mean age: $31 \pm 5.731$ ), the osteopenic group (mean age: $32.15 \pm 6.075$ ) and the osteoporotic group (mean age: $34.96 \pm 9.536)$ was found to be significant $(\mathrm{p}<0.000)$. Out of the total 1416 subjects who were included in the study, 1126 (79.5\%) were married, $268(18.6 \%)$ were single and $22(1.6 \%)$ were widows.

Out of the total 65 post-menopausal women who were included in the study, $32(49.3 \%)$ were found to be Osteoporotic, while 28 (43.1\%) were Osteopenic (as per WHO Criteria). In the Premenopausal women who were included in the study $(\mathrm{n}=1351), 17.8 \%$ were found to be Osteoporotic $(\mathrm{n}=240), 63.8 \%$ were reported to be Osteopenic $(\mathrm{n}=862)$ whole $18.4 \%$ individuals were found to be normal $(\mathrm{n}=249)$ (table I). Overall out of 1415 women, $272(19.2 \%)$ were found osteoporotic, 890 (62.9\%) were osteopenic and only 254 (17.9\%) were normal However, amongst all females family history of osteoporosis was found in 432 (30.5\%) women. (Table II).

The mean $\mathrm{T}$ score and standard deviation of Osteopenic and Osteoporotic females' were-1.826 \pm 0.539 and $2.93 \pm 0.73(\mathrm{p}<0.000)$ however $-0.567 \pm 0.417$, were for normal. The mean BUA scores were $92.38,75.66$ and 55.86 with estimated BMI 26.15, 24.030 and 23.59 ( $\mathrm{p}<0.624$ ) of normal, Osteopenia and Osteoporosis respectively shown in table III. Only $82(5.8 \%)$ women of all subjects did any physical exercise whereas the remaining 94.2\% women ( $\mathrm{n}=1331$ ) did not perform any exercise. Mean Intake of Calcium rich food per week was found to be 9.4 in normal individuals, 8.9 in Osteopenic females and 5.1 in those who were reported to have Osteoporosis.

\section{Discussion:}

Osteoporosis and low bone mass are major health problems throughout the world which are responsible for an increased risk of fractures. Fractures, such as hip fracture, are of greatest public health concern because the consequences are often devastating and may become a likeable cause of immobility and increased risk of death. According to National Osteoporosis foundation, a total of 44 million people in the United States of America were suffering from either Osteoporosis or low bone mass. 55\% of these Osteoporotic individuals included people with ages 50 and above. The Osteoporosis and the risk of developing the disease is expected to increase in more than 35 million women of age 50 and more in $2010^{[10]}$.

The present study shows the high prevalence of Osteoporosis and Osteopenia in Pakistani Pre and Postmenopausal women. These findings signify a high burden of these diseases in Pakistan. The diagnosis of Osteoporosis is done by BMD. BMD is an age-dependent variable thus showing an increasing trend in the prevalence of Osteoporosis with advancing age ${ }^{[11]}$. This aspect was also highlighted in our study.

With the overall increase in the population of Pakistan, the proportion of elderly and Post menopausal women is also increasing. The post-menopausal females are at a greater risk of developing Osteoporosis because very little or no estrogen is secreted by ovaries after menopause. This low estrogen level in the body results in increased bone loss. Studies have shown that estrogen causes apoptosis of osteoclastic cells and maintain an appropriate ratio between bone-forming osteoblasts and bone-resorbing osteoclasts ${ }^{[11,12]}$. Current study also shows that the frequency of Osteoporosis increases with increasing age $(\mathrm{p}<0.000)$.

There are several factors that can cause low bone mass in Premenopausal women such as low BMI. High BMI has been shown to have a protective effect on BMD ${ }^{[7]}$. However, insignificant differences of BMI were found in normal, Osteopenic and osteoporotic women in our study. Although an increased weight may have a positive impact on BMD, yet it also increases the risk of heart disease by increasing the cholesterol and causing diseases like diabetes, hypertension and stroke. A healthy weight decrease the risk of cancer of the breast, kidney, colon, and uterus. When all this is taken into account, the healthiest approach to protecting bones is not through weight gain but through other, healthier approaches, like exercise and a healthy diet.

Inadequate intake of nutrients and craze of losing weight may be a risk factor for dangerous conditions such as anorexia nervosa. It has been found in our study that those women who take more calcium rich foods on daily basis (especially dairy products) were protected from Osteoporosis. Decreased intake of calcium is a significant risk factor of Osteoporosis ${ }^{[7]}$ Calcium supplementation also has preventive effect against Osteoporosis but not many women take calcium supplements. Calcium supplementation also have preventive effect against Osteoporosis but very less women take calcium supplements i.e. only $26 \%$ women took calcium sometime or regularly may be due to improper counseling by physician. A nutritional counseling is required especially for pregnant and lactating women for the awareness of associated risk factors.

Weight-bearing exercises that work against gravity (like walking, stair climbing and weight training) help maintain strong bones. This research shows that $95 \%$ of Pakistani women don't do exercise (table II). Majority of the Pakistani women are house wives and thus spend a sedentary life style with minimum or no physical activity that makes them exposed to increased risk of heart disease, stroke, diabetes and obesity. 
Conner et al observed the influence of ethnicity on BMD and fractures and showed consistent ethnic differences in BMD such that blacks had the highest and Asians had the lowest BMD at all age groups ${ }^{[13]}$ because of their smaller body size. In this study all women were of Asian ethnicity due to which the association of ethnicity with Bone mineral Density could not be evaluated.

Smoking is another significant risk factor of Osteoporosis. Studies have shown lower prevalence of smoking in Pakistani females. However, there are several alternatives of smoking which are related with tobacco intake like "Paan", "Gutka" and "huqqa" (hubble bubble). We have excluded the females with history of tobacco intake in any form and thus have removed this important confounding factor ${ }^{[14]}$.

To diagnose Osteoporosis, calcaneal QUS was used in current study which has also been used by other studies to diagnose Osteoporosis. This technique is a reliable and cost effective method ${ }^{[15]}$. Our study shows high prevalence of Osteoporosis (19.2\%) and Osteopenia (62.9\%) as compared to the studies conducted in neighboring Indian and Korean women by using calcaneous QUS ${ }^{[16,17]}$.

Despite the advantages of the use of Quantitative ultrasound techniques, the DEXA scan is still considered as the Gold standard test for measuring BMD worldwide, thus limiting the reliability of the results of our study. This technology cannot accurately determine bone density but just gives a vague idea on whether the bone is healthy or not. If an ultrasound scan suspects reduced bone density, then the patient is advised to get a DEXA scan performed.

Another limitation of the study is that, collection of sample is limited to only one city, because of which the data collected during this study cannot describe the status of BMD of the whole country. Therefore, the results cannot be generalized on whole Pakistani population. However, the fact that subjects belonged to different towns of Karachi has somewhat replaced the need of door to door population based survey that are more appropriate and more representative of Population.

\section{Conclusion:}

The prevalence of osteoporosis was found to be high in postmenopausal but the incidence of Osteopenia in premenopausal women was reported to be alarmingly high in premenopausal women probably due to lifestyle and dietary risk. There is a need to explore the precise causative factors associated with the high prevalence of Osteopenia recorded in the present study.

\section{Acknowledgments:}

We greatly acknowledge BMD coordinators of Novartis Pharmaceuticals, Mr. Jahanzaib and Mr. Raza for helping us in data collection. We would also like to thank Mr. Stephen Dodd, Director Doddmed Ltd for providing us software of Sonost Sys 3000.We are also great full to Ms. Salina Mukhtar for reviewing and giving us important advices related to study

\section{References:}

[1] Neema, V. Shweta \& S. Inamdar. Prevalence of Osteoporosis using Quantitative Ultrasound for Menopausal women in Rural and Urban Area. The Internet Journal of Gynecology and Obstetrics. 2010; 13: 1

[2] Narang A., Arora S, Nagpal L. Osteoporosis and Homoeopathic Management. Posted April 2010.

[3] http://www.homeopathyworldcommunity.com/profiles/blogs/Osteoporosis-and-homoeopathic

[4] The Asian Audit: Epidemiology, costs and burden of Osteoporosis in Asia 2009 by International Osteoporosis foundation.

[5] http://www.iofbonehealth.org/publications/asian-audit-2009.html

[6] Gullberg B, Johnell O, Kanis JA.World-wide projections for hip fracture. Osteoporos Int 1997; 7: 407.

[7] Lubna. A Survey of Osteoporosis Risk Factors and Practices among Jordanian Women. Journal of International Women's Studies 2010 .

[8] http://www.faqs.org/periodicals/201005/2093689391.html

[9] Sultan A, Khan D A, Mushtaq M, Hassan MU. Frequency of Osteoporosis and its associated risk factors in postmenopausal women in clinical practice at Rawalpindi. Pak J Pathol. 2006; 17: 115-118

[10] Fatima M, Nawaz H, Kassi M, Rehman R, Kasi P M, Kassi M, Afghan A K, Baloch S N Determining the risk factors and prevalence of Osteoporosis using quantitative ultrasonography in Pakistani adult women. Singapore Med 2009; 50 (1) : 20

[11] Fahim F. The magnitude of low bone mineral [corrected] density in middle and old age women. J Pak Med Assoc 2005; 55:5002.Erratum in: J Pak Med Assoc 2006; 56:146.

[12] Nelson HD, Helfand M, Woolf SH, Allan JD. Screening for postmenopausal Osteoporosis: a review of the evidence for the U.S. Preventive Services Task Force. Ann Intern Med 2002; 137:529-41.

[13] http://www.nof.org/advocacy/resources/prevalencereport

[14] Ahlborg H G , Rosengren B E1, Järvinen T L N , Rogmark C , Jan-Åke Nilsson J A , Ingemar Sernbo I and Karlsson M K. Prevalence of Osteoporosis and incidence of hip fracture in women - secular trends over 30 years2010 BMC Musculoskeletal Disorders, 11:48doi:10.1186/1471-2474-11-48

[15] Krum SA, Miranda-Carboni G A,Hauschka P V, Carroll J S, Lane T F, Freedman L P and Brown M. Estrogen protects bone by inducing Fas ligand in osteoblasts to regulate osteoclasts survival. The EMBO Journal (2008) 27, $535-545$.

[16] Connor E B, Siris E S, Wehren L E, Miller P D, Abbott T A, Berger M L, Thomas A Arthur C, Santora T A A, Sherwood L M. Osteoporosis and Fracture Risk in Women of Different Ethnic Groups. Journal of Bone and Mineral Research 2005; 20: 185-194

[17] Khan JA, Hussain SF, Malik A, et al. Knowledge, attitudes and the prevalence of smoking among hospital attendants in a developing country. Trop Doct 2003; 33:231-4. 
[18] Thuy VTT, Chau TT, Cong ND, et al. Assessment of low bone mass in Vietnamese: comparison of QUS calcaneal ultrasonometer and data-derived T-scores. J Bone Miner Metab 2003;21:114-19. (s)

[19] Sharma S,Tandon VR, Mahajan A, et al. Preliminary screening of Osteoporosis and Osteopenia in urban women from Jammu using calcaneal QUS. Indian J Med Sci 2006; 60:183-9.

[20] Kim CH, Kim YI, Choi CS, et al. Prevalence and risk factors of low quantitative ultrasound values of calcaneus in Korean elderly women. Ultrasound Med Biol 2000; 26:35-40.

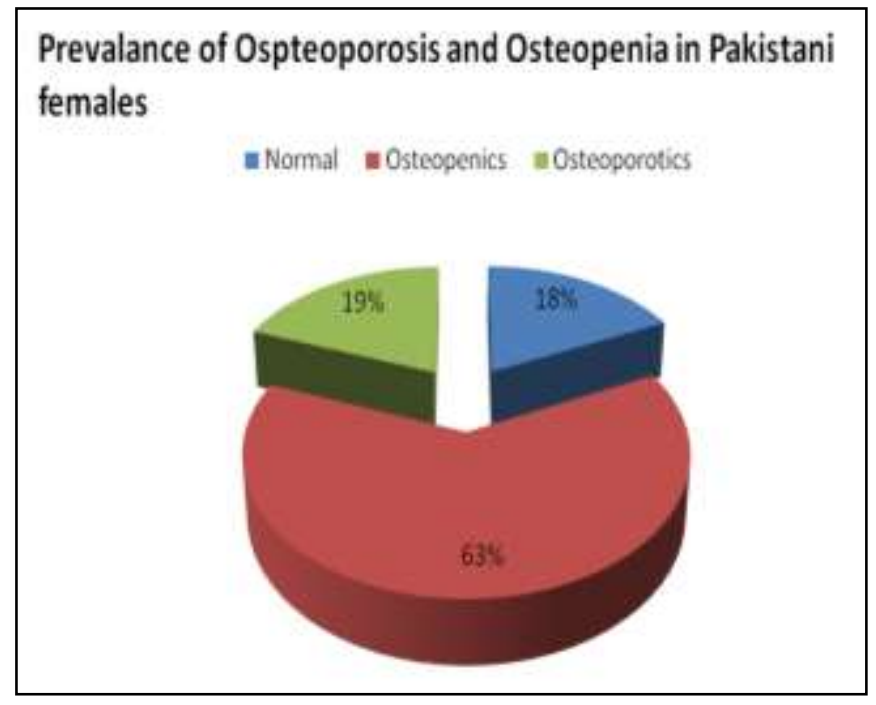

Figure III

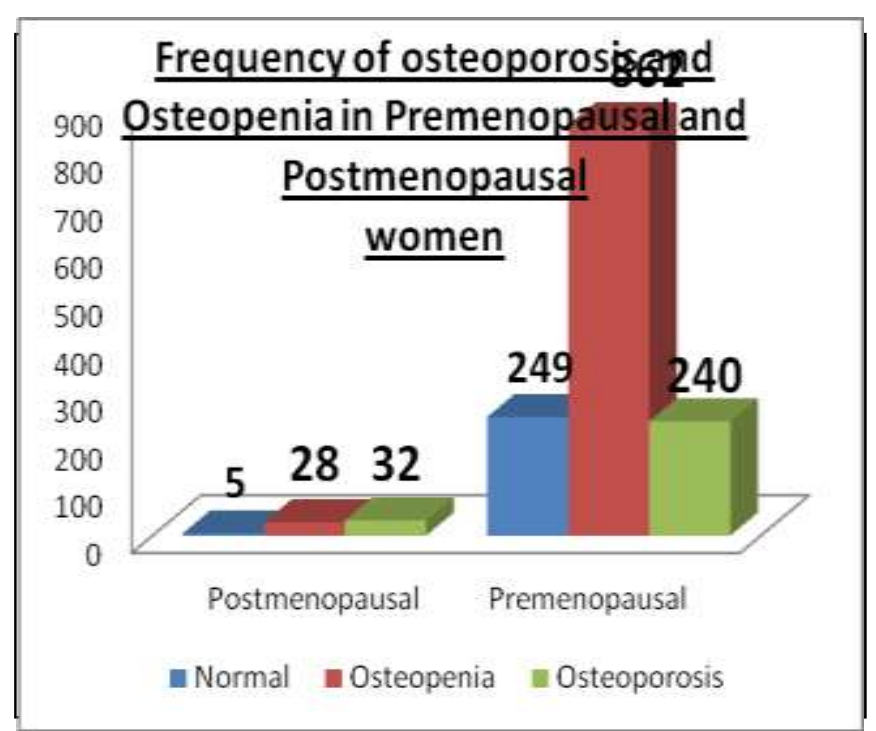

Table I: Bone Mineral Density in Premenopausal and Postmenopausal women

\begin{tabular}{|c|l|l|l|l|}
\hline & \multicolumn{4}{|c|}{ Bone Mineral Density (BMD) } \\
\cline { 2 - 5 } & Normal & Osteopenia & $\begin{array}{l}\text { Osteoporosi } \\
\text { s }\end{array}$ & Total \\
\hline & & & & \\
\hline $\begin{array}{l}\text { Menstrual Status } \\
\text { Postmenopausal }\end{array}$ & $5(7.7 \%)$ & $28(43.1 \%)$ & $32(49.3 \%)$ & $65(100 \%)$ \\
\hline Premenopausal & $249(18.4 \%)$ & $862(63.8 \%)$ & $240(17.8 \%)$ & $1351(100 \%)$ \\
\hline Total & $254(17.9 \%)$ & $890(62.9 \%)$ & $272(19.2 \%)$ & $1416(100 \%)$ \\
\hline & & & & \\
\hline
\end{tabular}


Table II: Demographic characteristics of the population studied

\begin{tabular}{|l|c|c|}
\hline \multicolumn{1}{|c|}{ Variable } & Frequency & Percentage (\%) \\
\hline Marital Status & & \\
\hline Single & 268 & 18.6 \\
\hline Married & 1126 & 79.5 \\
\hline Widowed & 22 & 1.6 \\
\hline & & \\
\hline Menstrual History & 65 & 4.6 \\
\hline Pre menopausal & 1351 & 95.4 \\
\hline Post menopausal & & \\
\hline & & 95 \\
\hline Exercise (min/day) & 1345 & 5 \\
\hline Yes & 71 & \\
\hline No & & 8.5 \\
\hline & & 91.5 \\
\hline Ca Supplementation & 120 & \\
\hline yes & 1296 & 30.5 \\
\hline No & & \\
\hline & & \\
\hline Family History of Osteoporosis & 432 & \\
\hline Yes & 984 & \\
\hline No & & \\
\hline & & \\
\hline
\end{tabular}

Table III: Mean values and standard deviation of the variables

\begin{tabular}{|c|c|c|c|c|}
\hline Mean Value & Normal & Osteopenia & Osteoporotic & P value \\
\hline T Score & $-0.567 \pm 0.417$ & $-1.826 \pm 0.539$ & $-2.93 \pm 0.734$ & $<0.001$ \\
\hline SOS & $151.94 \pm 1.218$ & $150.09 \pm 1.12$ & $148.18 \pm 1.34$ & $<0.026$ \\
\hline BUA & $92.38 \pm 13.708$ & $75.66 \pm 12.827$ & $55.86 \pm 14.419$ & $<0.000$ \\
\hline BMI(kg/m $\left.{ }^{2}\right)$ & $26.150 \pm 2.719$ & $24.030 \pm 7.7$ & $23.59 \pm 2.29$ & $<0.624$ \\
\hline Age(years) & $31.06 \pm 5.731$ & $32.16 \pm 6.075$ & $34.96 \pm 9.536$ & $<0.000$ \\
\hline $\begin{array}{c}\text { Calcium } \\
\text { Intake }\end{array}$ & $9.6 \pm 0.78$ & $8.9 \pm 2.5$ & $5.1 \pm 8.5$ & $<0.04$ \\
\hline
\end{tabular}

